\title{
The effects of impurities and core pressure on pedestal stability in Joint European Torus (JET) ${ }^{\text {a) }}$
}

\author{
S. Saarelma, ${ }^{1, b), c)}$ A. Järvinen, ${ }^{2}$ M. Beurskens, ${ }^{1}$ C. Challis, ${ }^{1}$ L. Frassinetti, ${ }^{3}$ C. Giroud, ${ }^{1}$ \\ M. Groth, ${ }^{2}$ M. Leyland, ${ }^{4}$ C. Maggi, ${ }^{1}$ J. Simpson, ${ }^{1}$ and JET Contributors ${ }^{\text {d) }}$ \\ EUROfusion Consortium JET Culham Science Centre, Abingdon OX14 3DB, United Kingdom \\ ${ }^{1}$ Culham Centre for Fusion Energy, Culham Science Centre, Abingdon OX14 3DB, United Kingdom \\ ${ }^{2}$ Aalto University, Otakaari 4, 02150 Espoo, Finland \\ ${ }^{3}$ Department of Fusion Plasma Physics, School of Electrical Engineering, KTH Royal Institute of Technology, \\ SE-10044 Stockholm, Sweden \\ ${ }^{4}$ York Plasma Institute, Department of Physics, University of York, Heslington, York YO10 5DD, United Kingdom
}

(Received 14 November 2014; accepted 23 April 2015; published online 21 May 2015)

The H-mode pedestal plays an important role in determining global confinement in tokamaks. In high triangularity H-mode experiments in Joint European Torus with the ITER-like wall (JET-ILW), significantly higher pedestal temperature and global confinement have been achieved with nitrogen seeding. The experimentally observed increase in pedestal height is inconsistent with the stability calculations using the experimental profiles. Numerically, we find that the consistency with stability improvement can be restored if we assume a shift of the pedestal inwards and increased ion dilution due to the impurity seeding. Significantly better confinement and pedestal height have been observed in JET-ILW plasmas when the core pressure is increased. The enhanced pedestal height can be linked to an improvement in edge stability arising from an increase in the Shafranov-shift, higher edge current, and pedestal widening in flux space. [http://dx.doi.org/10.1063/1.4921413]

\section{INTRODUCTION}

The global plasma confinement in a tokamak operating in high confinement or H-mode is largely determined by the edge pedestal pressure due to core turbulence restricting the temperature gradient near the marginal stability limit. ${ }^{1}$ The transport simulations using the trapped gyro-Landau fluid (TGLF) transport model show that the ITER fusion power scales like $T_{\text {ped }}^{2},{ }^{2}$ where $\mathrm{T}_{\text {ped }}$ is the temperature at the top of the pedestal. The pedestal of Type I ELMy H-mode (the typical operation regime of current tokamaks and the baseline operation for ITER) has been shown to be limited by ideal MHD peelingballooning modes in Joint European Torus (JET), ${ }^{3}$ DIII$\mathrm{D}^{4}{ }^{\mathrm{JT}} \mathrm{-60U},{ }^{5}$ and ASDEX Upgrade. ${ }^{6}$ Therefore, if the edge peeling-ballooning stability can be improved, higher pedestals and, consequently, better global plasma performance can be achieved. The shaping of plasma, especially increasing triangularity, has been shown experimentally and numerically to lead to higher pedestals. ${ }^{4}$ However, this method has not been very successful in Joint European Torus with the ITER-like wall (JET-ILW) baseline experiments, where no difference in confinement between low- and high-triangularity was observed. ${ }^{7}$ This paper investigates at two other possible mechanisms to increase the pedestal height to improve the performance: increasing core pressure and seeding of low-Z impurities.

\footnotetext{
${ }^{\text {a)}}$ Paper BI1 1, Bull. Am. Phys. Soc. 59, 22 (2014).

${ }^{\text {b) Invited speaker. }}$

c)samuli.saarelma@ccfe.ac.uk

d) See the Appendix of F. Romanelli et al., Proceedings of the 25th IAEA Fusion Energy Conference, St. Petersburg, Russia, 2014.
}

\section{ANALYSIS METHOD}

In this paper, we analyse the pedestal stability of plasma equilibria. First, we recreate the investigated equilibrium using the pedestal temperature and density profiles measured using the Thomson scattering systems..$^{8-10}$ The profiles are fitted with the modified tanh-function described in Ref. 11. Since the standard equilibrium reconstruction is usually not accurate enough to give the separatrix location in relation to the profiles, we have to radially shift the measured profiles to be consistent with the power balance at the separatrix. In this paper, we use a simple two-point model $^{12}$ to determine the separatrix electron temperature $\mathrm{T}_{\mathrm{e} \text {,sep }}$ when there are no significant factors within an investigated scan to create differences between plasmas and more complete multi-fluid modelling using the EDGE2D-EIRENE code ${ }^{13-15}$ when we assume that $\mathrm{T}_{\mathrm{e} \text {,sep }}$ is affected by changes in the 2-D characteristics of the scrape-off layer. Since the density and temperature profiles are measured by the same diagnostic, both are shifted by the same amount.

We assume that the current profile is composed of bootstrap and fully diffused Ohmic currents. The bootstrap current is calculated self-consistently using the experimental density and temperature profiles and formulas in Ref. 16. The Ohmic current profile shape follows the neo-classical conductivity profile (calculated from the density and temperature profiles) and its amplitude is adjusted so that the total current agrees with the experimental value. To find the stability boundaries, we either perturb the normalised pedestal pressure gradient $\left(\alpha=\left(2 \partial_{\psi} V\right) /(2 \pi)^{2}\left(\frac{V}{2 \pi^{2} R}\right)^{\frac{1}{2}} \mu_{0} p^{\prime}\right.$, where $V$ is the volume enclosed by the flux surface, $R$ is the major radius and $p^{\prime}$ is the pressure derivative with respect to the poloidal flux $\psi$ ) and current density (j) from the values of the 
experimental equilibrium to produce a $\mathrm{j}-\alpha$ diagram showing the stability boundaries in relation to the investigated equilibrium or we vary the temperature pedestal height and calculate the current profile self-consistently to find the marginally stable temperature pedestal height. Whilst the former method gives us a general picture of the stability around the investigated plasma, it is not always very good for determining the distance from the marginal stability. On the other hand, the selfconsistent method provides us a scalar value for the marginal stability, which can then be compared with the experimental pedestal height or other stability analysis results. In the stability calculations, we use the ideal MHD stability code ELITE. ${ }^{17}$

\section{POWER SCAN}

To study the connection between the core pressure and the pedestal stability, a power scan JET experiment at $\mathrm{I}_{\mathrm{p}}=1.4 \mathrm{MA}$ and $\mathrm{B}_{\mathrm{t}}=1.7 \mathrm{~T}$ using neutral beam injection (NBI) only was conducted at low $\left(\delta_{\mathrm{u}}=0.2\right)$ and high $\left(\delta_{\mathrm{u}}=0.4\right)$ triangularities. The NBI heating power was varied between 4 and $15 \mathrm{MW}$. The gas injection rate was kept as low as possible while still ensuring high enough Edge Localised Mode (ELM) frequency to avoid high tungsten influx and radiation. The power degradation in the experiment was much weaker than the IPB98(y,2) scaling. There are several factors that contribute to the good confinement at high power (density peaking at low collisionality, longer fast ion slowing down time resulting higher fast ion pressure and improved core turbulence suppression due to electromagnetic effects), but here we concentrate only on the confinement improvement due to the increased pedestal height by improved pedestal stability. From the point of view of the pedestal, all the effects of increased core confinement and heating can be grouped together into an increased core pressure profile characterised by global plasma $\beta_{\mathrm{n}}$. The range of $\beta_{\mathrm{N}}$ in the experiment was 1.5-3. More detailed description of the experiment is given in Ref. 18. In the equilibrium reconstruction, we used the density and temperature profiles that were in the last $30 \%$ of the ELM cycle. The change of the $\mathrm{j}-\alpha$ stability diagram at high triangularity as $\beta_{\mathrm{N}}$

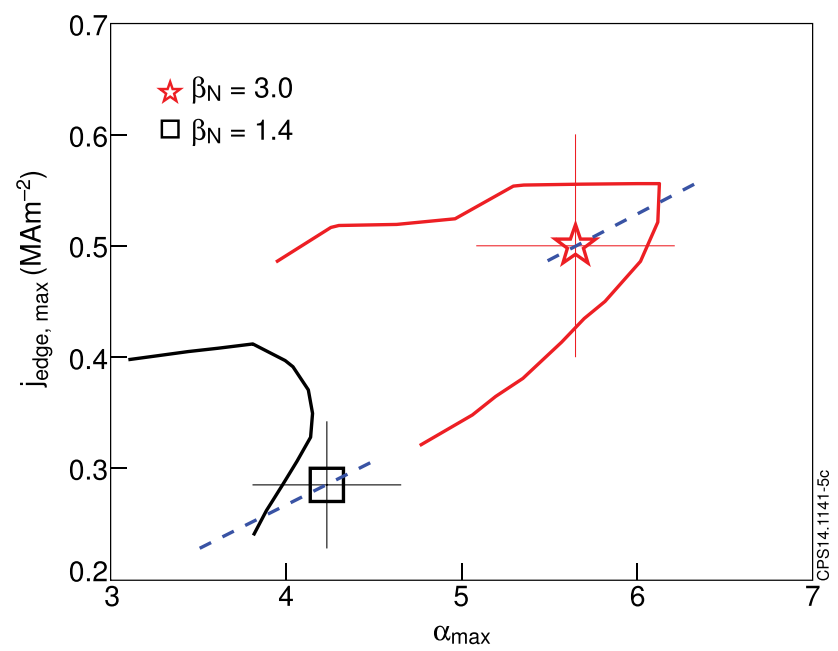

FIG. 1. Stability boundaries and operational points of the lowest and the highest $\beta_{\mathrm{n}}$ values of the high $\delta$ power scan. The dashed lines show the selfconsistent path with varying $\mathrm{T}_{\text {ped. }}$. is increased is shown in Figure 1. For both low (1.5) and high $\beta_{\mathrm{N}}(3.0)$, the experimental equilibria are close to their respective stability boundaries. In the self-consistent analysis (vary the pedestal height from the experimental value to find the marginally stable pedestal height), we find that all the plasmas in the power scan are within measurement error from their stability limit. At low power, there is no difference between low and high triangularity plasmas, but as the power is increased, the high triangularity plasmas see larger increase both in measured and modelled pedestal height. The increase in marginally stable $\alpha$ between the low and high $\beta_{\mathrm{N}}$ cases is only about $50 \%$ (Fig. 2), while the pedestal height more than doubles. This is explained by the pedestal widening with increasing $\beta_{\mathrm{N}}$ allowing the pedestal height to increase without a corresponding increase in the gradient. The pedestals widen only in flux space. In real space (at the outboard midplane), the width does not increase, so the widening in flux space can be associated with the increasing Shafranov-shift that pushes the flux surfaces closer together on the outboard side. The self-consistent marginally stable pedestal height is also increased by decreasing pedestal collisionality that leads to higher bootstrap current. Higher edge current allows access to the "nose" of stability diagram and so achieves high values of $\alpha$ without triggering the limiting peeling-ballooning modes. In Fig. 1, the self-consistent path of the $\beta_{\mathrm{N}}=3.0$ case crosses the stability boundary at the maximum value of $\alpha$ allowed by the stability boundary, while the $\beta_{\mathrm{N}}=1.4$ becomes limited at $\alpha=3.8$ even though the maximum $\alpha$ allowed by the stability boundary at higher current density would be 4.1 .

It should be noted that the stability improvement with increasing core pressure is only seen with low gas injection rates. When the gas injection is increased, the beneficial effect of the core pressure is strongly reduced. ${ }^{19}$ It is not entirely clear why the pedestal improvement by high core pressure is lost at high gas rates, but it would be consistent with the stability picture at low pedestal current density at which part of the stability diagram the improvement due to

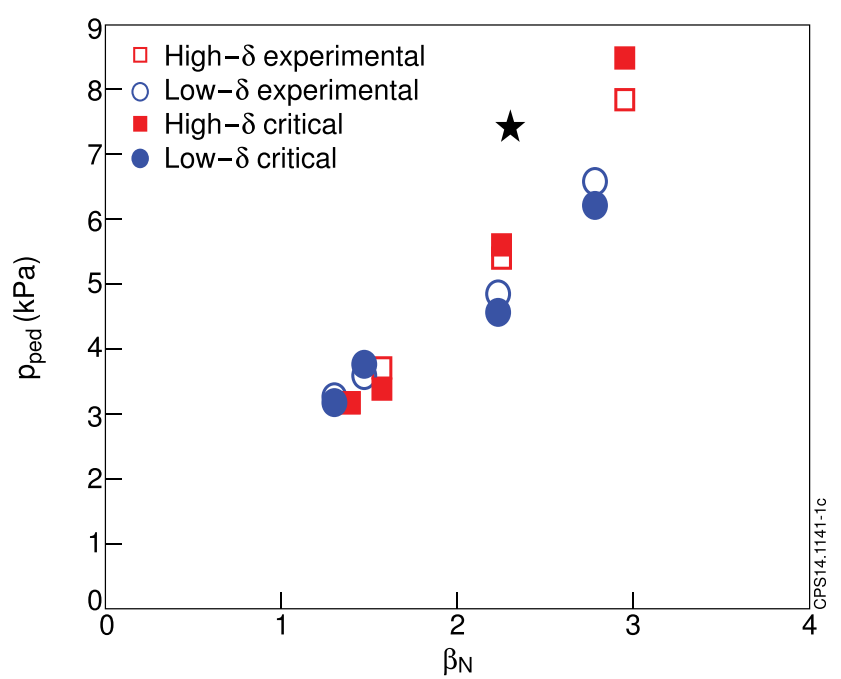

FIG. 2. The measured and self-consistently modelled pedestal heights as a function of global $\beta_{\mathrm{N}}$. The black star shows the critical pedestal height for high triangularity case of $\beta_{\mathrm{n}}=2.3$ calculated using the pedestal widths of the $\beta_{\mathrm{n}}=3.0$ case 
increased $\beta_{\mathrm{N}}$ is reduced. In a recent paper by Belli et al. ${ }^{20}$ it was shown that the bootstrap current calculations are overestimated by using Sauter's formula ${ }^{16}$ if the collisionality is high. If this is indeed the case with the high gas cases (that have higher collisionality than the low gas cases), they can become consistent with the peeling-ballooning modes limiting the pedestal height. At lower edge current density, the peeling-ballooning boundary is reached at considerably lower value than at high edge current density (as is can be seen in Fig. 1).

\section{NITROGEN SEEDING}

In JET with Be/W wall (ITER-like-wall or JET-ILW) high triangularity baseline plasmas $\left(\beta_{\mathrm{N}}<1.5\right)$ operating at high gas fuelling to avoid tungsten influx, the confinement has been degraded by $40 \%$ compared with earlier experiments with carbon wall (JET-C) with same heating power and density. ${ }^{21}$ The lower confinement is mainly due to lower pedestal temperature. ${ }^{22}$ However with nitrogen seeding, the pedestal and the global confinement can be partially recovered. The confinement improvement with nitrogen seeding is more modest in low triangularity. ${ }^{21}$ In JET-C, the dominant impurity in the edge region was carbon, leading to $\mathrm{Z}_{\mathrm{eff}} \approx 2.0$ (with $\mathrm{n}_{\mathrm{i}} / \mathrm{n}_{\mathrm{e}}=0.8$ ), whereas in JET-ILW without impurity seeding, the main impurity is beryllium, leading to $\mathrm{Z}_{\mathrm{eff}} \approx 1.2$ (Ref. 21) (with reduced ion dilution of $n_{i} / n_{e}=0.93$ ). In nitrogen seeded discharges, nitrogen becomes the main impurity and $Z_{\text {eff }}$ increases to similar level as in JET-C (Ref. 21). The uncertainty in the $Z_{\text {eff }}$ measurements is about $20 \%$.

We first compare the stability of the unseeded and nitrogen seeded experimental JET-ILW plasmas using the actual experimental pressure profiles (shown in Figure 5) and the two-point model for the needed radial shift of the profiles, which gives $100 \mathrm{eV}$ for both cases. The resulting stability diagram is shown in Figure 3. The stability boundaries for the two cases are almost the same. This is a combination of two factors. On one hand, the nitrogen seeded case has a wider

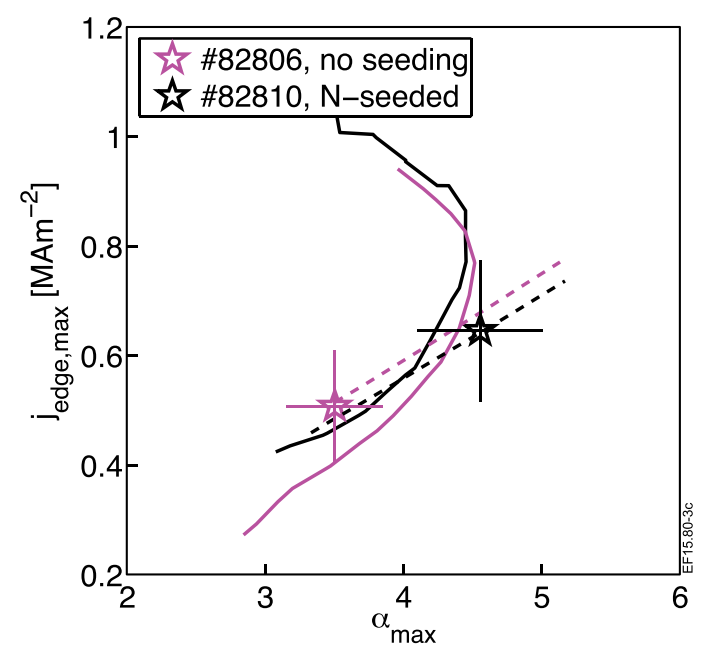

FIG. 3. The stability boundaries of the unseeded (\#82806) and nitrogen seeded (\#82810) experimental JET plasmas (solid lines). The stars show the location of the experimental pedestal and the dashed lines show the selfconsistent track of the experimental point as the temperature pedestal height is increased. pedestal. This is destabilising for the same $\alpha_{\max }$. On the other hand, the maximum gradient location of the unseeded is closer to the plasma edge. This is also destabilising for the same $\alpha_{\max }$. Together these two effects almost cancel each other. We can also see that although the experimental points in the diagram are quite far from each other, the selfconsistent paths are almost identical and they cut the stability boundaries almost at the same place. Thus, the self-consistent critical pedestal pressure in the two cases is almost the same ( $16 \mathrm{kPa}$ for the unseeded and $15 \mathrm{kPa}$ for the seeded), while the experimental pedestal height of the seeded case is significantly higher ( 21 vs. $13 \mathrm{kPa})$.

From this analysis, we can conclude that either something else than the peeling-ballooning modes are limiting the unseeded case pedestal to a lower pedestal pressure, some mechanism not captured by the bootstrap current formula used in this analysis lowers the unseeded case pedestal current making it high-n ballooning mode limited at lower value of $\alpha_{\max }$ (but does not affect the seeded ca case) or there is some other mechanism that moves the stability boundaries. Such a possible mechanism based on the lowered separatrix temperature with impurity seeding combined with ion dilution is discussed next.

One of the major differences between JET-ILW without seeding and JET-C plasmas is the lower radiation in the divertor region due to the lack of low- $\mathrm{Z}$ impurities, such as carbon. ${ }^{21}$ Nitrogen seeding allows return to the divertor radiation level similar to JET-C. The multi-fluid code EDGE2DEIRENE was executed for steady-state, inter-ELM simulations for these plasmas. ${ }^{23,24}$ The radial transport coefficients for heat conduction and particle diffusion were adjusted to reproduce the experimentally measured electron density and temperature profiles at the low-field side mid-plane in the unseeded reference plasmas in JET-ILW. ${ }^{22}$ Based on this base case, nitrogen injection from low levels of radiation to the maximum radiation levels reached in the simulations were conducted without changing any other boundary conditions in the simulations. When doing this, the simulations do reproduce the overall divertor radiation levels observed in the experiment with nitrogen seeding, whereas in the unseeded base case, the divertor radiation levels were underestimated by a factor of two. ${ }^{22}$ With increasing nitrogen seeding and divertor radiation, the simulations show a robust increase in the pedestal neutral fuelling caused by recycling neutrals penetrating through the divertor plasma. This occurs due to increased transparency of the divertor plasma to recycling neutrals as the nitrogen radiation cools down the scrape-off-layer (SOL) plasma in the low field side (LFS) divertor leg. This leads to increased electron densities and convective heat transport fraction at the LFS mid-plane separatrix, which both reduce the separatrix electron temperatures in high recycling plasma solutions, in addition to the residual nitrogen radiation inside the separatrix. The impact of these mechanisms on the separatrix temperature is typically about $5-15 \mathrm{eV}$ within the sensitivity scans conducted in these studies. In plasma solutions that are detached in the LFS divertor leg, the nitrogen radiation zone shifts partially inside the separatrix, reducing the power crossing the separatrix. This can provide further about $10-20 \mathrm{eV}$ reduction of 
the separatrix temperature, in agreement with the simple two-point model arguments. However, there is an obvious shortcoming in this EDGE2D-EIRENE analysis. Since the radial transport coefficients are not changed in the simulated scan, the simulations are not able to reproduce the experimentally observed increase in pedestal temperatures with nitrogen seeding. Nevertheless, sensitivity scans indicate that the experimentally observed pedestal temperatures can be obtained in the simulations by scaling the radial heat conductivity values inside the separatrix down by a factor of 2-3 with a negligible impact on the SOL plasma solution. This gives confidence that the SOL solution can indeed be decoupled from the physics details occurring inside the pedestal. Therefore, the obtained reduction of separatrix electron temperature at the LFS mid-plane may not be completely unphysical, if it is assumed that the details of the 2-D plasma and neutral transport characteristics of the SOL, which the two-point model cannot capture, have a significant impact on the electron temperature and density distributions in the SOL, as they do in the simulations. However, none of the 2D multi-fluid codes carries the predictive capability for the pedestal profiles, and, therefore, the obtained reduction of the separatrix temperature may be over or underestimated or even the scaling of the separatrix electron temperature with nitrogen radiation may not be captured appropriately. Therefore, these results are used here to provide an educated estimate for the possible reduction of the separatrix electron temperature with nitrogen seeding, understanding that this estimate may not entail the entire set of physics occurring in the actual experimental situation. In the following analysis, we assume a $20 \mathrm{eV}$ drop in $\mathrm{T}_{\mathrm{e} \text {,sep }}$ as a result of seeding nitrogen. The purpose of the following stability analysis is to explore the qualitative trends that the drop in separatrix temperature and the consequent shift of the profiles have on the marginal peeling-ballooning mode stability of the pedestal, not to reproduce the observed pedestal increase exactly.

To test the effect of $\mathrm{T}_{\mathrm{e} \text {,sep }}$ on the stability, we use an unseeded JET discharge \#82 806, $\left(\mathrm{B}_{\mathrm{t}}=2.7 \mathrm{~T}, \mathrm{I}_{\mathrm{p}}=2.5 \mathrm{MA}\right.$, $\left.\beta_{\mathrm{N}}=1.1\right)$. We vary the separatrix temperature and shift the profiles accordingly. The effect on the low $(\delta=0.2)$ and high $(\delta=0.4)$ triangularity stability boundaries is shown in Figure 4. The main difference in the stability boundaries is in the high- $\alpha$ part at high current where the intermediate-n peelingballooning modes are the limiting instability, while the low current part with high-n ballooning modes is hardly changed by the shift of the profiles. The small improvement in the ballooning stability agrees with the local $n=\infty$ ballooning mode stability, which does not change at all with the shift. It should also be noted that the stability improvement is larger for the high triangularity case. In a self-consistent analysis, we find an increase in $10 \%$ in critical temperature pedestal height for a decrease in $10 \%$ in $\mathrm{T}_{\mathrm{e}, \mathrm{sep}}$.

We also vary the charge number of the impurity $\left(Z_{\mathrm{imp}}\right)$ with fixed $Z_{\text {eff }}$ and $T_{e, s e p}$ in the simulation to determine the effect of different impurities on the pedestal stability. $\mathrm{Z}_{\mathrm{imp}}$ enters the equilibrium reconstruction in two ways: Low $\mathrm{Z}_{\mathrm{imp}}$ leads to more ion dilution and, thus, lower ion density $n_{i}$ and total pressure gradient; and impurities with low $\mathrm{Z}_{\mathrm{imp}}$ also reduce the bootstrap current more than high $\mathrm{Z}_{\mathrm{imp}}$ impurities

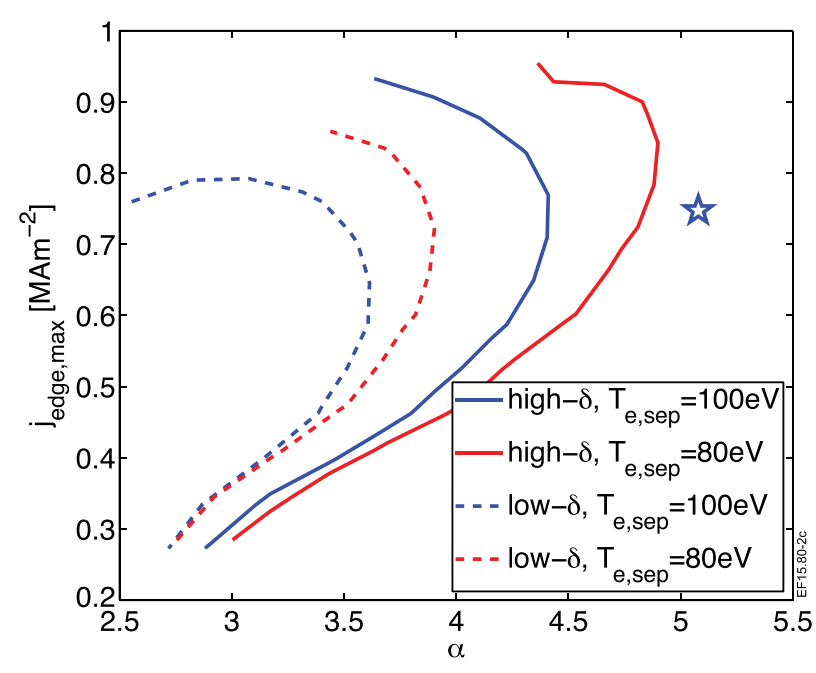

FIG. 4. The effect of the assumed separatrix temperature on stability boundaries for low (dashed) and high (solid) triangularities.

for a given value of $\mathrm{Z}_{\text {eff }}$. When these effects are combined in a self-consistent simulation, we see a monotonic decrease in marginally stable $\mathrm{T}_{\text {e,ped }}$ as a function of $\mathrm{Z}_{\text {imp }}$ of about $5 \%$ between beryllium $\left(Z_{i m p}=4\right)$ and neon $\left(Z_{i m p}=10\right)$. Since the marginally stable $T_{\text {e,ped }}$ decreases with $Z_{\text {imp }}$, the seeding with impurities that have higher $\mathrm{Z}$ than nitrogen is unlikely to be beneficial unless it can reduce $\mathrm{T}_{\mathrm{e} \text {,sep }}$ further. These impurities also radiate more strongly in the pedestal and the core than nitrogen, which is also detrimental to reaching high $\mathrm{T}_{\mathrm{e} \text {,ped }}$.

We combine all the effects and take into account expected pedestal widening as the height increases with the relation: $\Delta \sim \sqrt{\boldsymbol{\beta}_{\text {p,ped }}}$ ( $\Delta$ is the width of the pedestal and $\beta_{\mathrm{p}, \mathrm{ped}}$ is the poloidal $\beta$ at the top of the pedestal). This relation is used in a predictive EPED1 model $^{25}$ and seen to be valid in JET-C (although not distinguishable from constant real space width combined with flux compression) ${ }^{26}$ and in JET-ILW with nitrogen seeding. ${ }^{27}$ Table I shows the result of the self-consistent analysis at fixed $\mathrm{Z}_{\text {eff }}=1.5$ for beryllium $\left(\mathrm{n}_{\mathrm{i}} / \mathrm{n}_{\mathrm{e}}=0.875\right)$ and nitrogen $\left(\mathrm{n}_{\mathrm{i}} / \mathrm{n}_{\mathrm{e}}=0.929\right)$ impurities for two separatrix temperatures, 80 and $100 \mathrm{eV}$. The marginally stable pedestal profiles for all the four cases are shown in Fig. 5. Beryllium as an impurity reaches higher pedestal for both assumed values of $\mathrm{T}_{\mathrm{e}, \mathrm{sep}}$. However, as was shown in Figure 3 , it is the nitrogen seeding that leads to lower $\mathrm{T}_{\mathrm{e} \text {,sep }}$. Therefore, the comparable modelled marginally stable pedestals are nitrogen at $\mathrm{T}_{\mathrm{e}, \mathrm{sep}}=80 \mathrm{eV}$ and beryllium at

TABLE I. Marginally stable pedestal top pressures for the two impurity species $(\mathrm{Be}$ and $\mathrm{N}$ ) and two assumed separatrix temperatures (80 and $100 \mathrm{eV}$ ). The experimental values of the seeded and the unseeded cases are shown as well.

\begin{tabular}{lcc}
\hline \hline & $\begin{array}{c}\text { Nitrogen seeded, } \\
\text { Zimp }=7\end{array}$ & $\begin{array}{c}\text { Beryllium as main } \\
\text { impurity, Zimp }=4\end{array}$ \\
\hline $80 \mathrm{eV}$ & $16.7 \mathrm{kPa}$ & $20.0 \mathrm{kPa}$ \\
$100 \mathrm{eV}$ & $9.6 \mathrm{kPa}$ & $10.9 \mathrm{kPa}$ \\
Experimental & $21.0 \mathrm{kPa}(\# 82810)$ & $12.7 \mathrm{kPa}(\# 82806)$ \\
\hline \hline
\end{tabular}




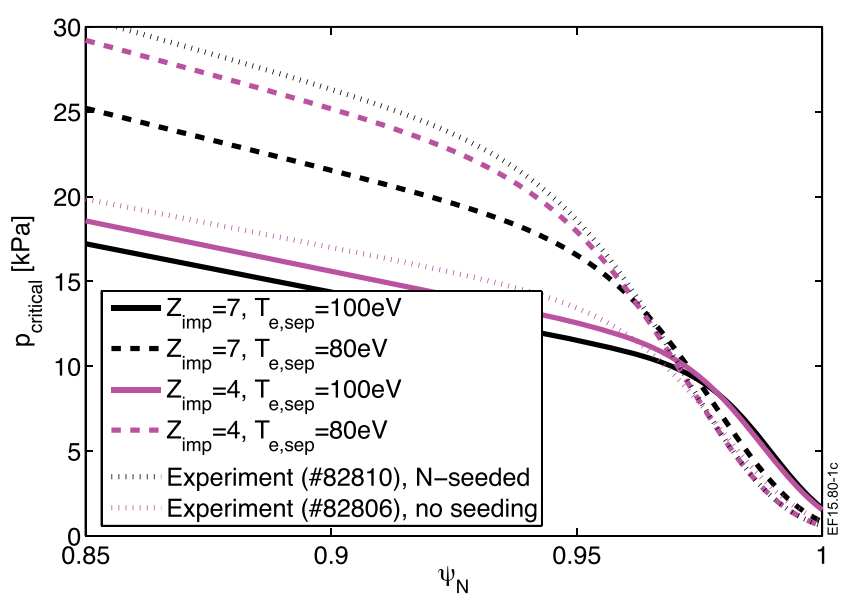

FIG. 5. The marginally stable edge pressure profiles for the four cases shown in Table I as well as the experimental pressure profiles for the nitrogen seeded and unseeded plasmas.

$\mathrm{T}_{\mathrm{e}, \mathrm{sep}}=100 \mathrm{eV}$. We can see that the pedestal stability modelling at least qualitatively captures the experimental pedestal height difference between the seeded and the unseeded cases.

\section{CONCLUSIONS}

The experimentally observed increases of pedestal height with increasing core pressure and impurity content in the pedestal region are consistent with improved stability in ideal MHD peeling-ballooning mode modelling of the pedestal. In the JET power scan experiment, the increased pedestal height is in part due to an increased stability limit for the pressure gradient by the increased Shafranov-shift and the accessing the "nose" of the stability diagram and in part due to pedestal widening that leads to a higher pedestal for given gradient. The comparison of the stability of a nitrogen seeded and unseeded experimental plasmas does without assuming other effects affecting the profiles or the bootstrap current shows that the increased pedestal height in the seeded plasma is inconsistent with the paradigm of peeling-ballooning modes limiting the pedestal. Only if we assume that the profiles are shifted inwards due to the seeding improving the pedestal stability, the consistency between the experiment and the stability analysis can be achieved.

The stabilising effect of the core pressure on the pedestal shown experimentally and in stability analysis in this paper leads to a feedback loop with increased pedestal height improving the core confinement (as predicted in Ref. 25 and seen also in wider JET dataset ${ }^{28}$ ), and are partly responsible for the very favourable power scaling found in JET. ${ }^{18}$ The possibility of affecting the pedestal stability (and, through that, plasma confinement) with impurities opens new possibilities for future devices that are in any case going to have to radiate a large fraction of the fusion power using extrinsic impurity seeding. However, more modelling and experiments are needed to fully understand the effects the impurities have on the pedestal and to optimise the right impurity seeding for each device.

\section{ACKNOWLEDGMENTS}

This work has been carried out within the framework of the EUROfusion Consortium and has received funding from the European Union's Horizon 2020 research and innovation programme under Grant Agreement No. 633053 and from the RCUK Energy Programme [Grant No. EP/I501045]. To obtain further information on the data and models underlying this paper, please contact PublicationsManager@ccfe.ac.uk. The views and opinions expressed herein do not necessarily reflect those of the European Commission.

${ }^{1}$ F. Ryter, C. Angioni, M. Beurskens, S. Cirant, G. T. Hoang, G. M. D. Hogeweij, F. Imbeaux, A. Jacchia, P. Mantica, W. Suttrop, and G. Tardini, Plasma Phys. Controlled Fusion 43, A323 (2001).

${ }^{2}$ J. E. Kinsey, G. M. Staebler, J. Candy, R. E. Waltz, and R. V. Budny, Nucl. Fusion 51, 083001 (2011).

${ }^{3}$ S. Saarelma, A. Alfier, M. N. A. Beurskens, R. Coelho, H. R. Koslowski, Y. Liang, I. Nunes, and JET EFDA Contributors, Plasma Phys. Controlled Fusion 51, 035001 (2009).

${ }^{4}$ P. B. Snyder, H. R. Wilson, J. R. Ferron, L. L. Lao, A. W. Leonard, D. Mossessian, M. Murakami, T. H. Osborne, A. D. Turnbull, and X. Q. Xu, Nucl. Fusion 44, 320 (2004).

${ }^{5}$ G. Saibene, N. Oyama, J. Lönnroth, Y. Andrew, E. de la Luna, C. Giroud, G. T. A. Huysmans, Y. Kamada, M. A. H. Kempenaars et al., Nucl. Fusion 47, 969 (2007).

${ }^{6}$ P. B. Snyder, N. Aiba, M. Beurskens, R. J. Groebner, L. D. Horton, A. E. Hubbard, J. W. Hughes, G. T. A. Huysmans, Y. Kamada, A. Kirk et al., Nucl. Fusion 49, 085035 (2009).

${ }^{7}$ M. N. A. Beurskens, L. Frassinetti, C. Challis, C. Giroud, S. Saarelma, B. Alper, C. Angioni, P. Bilkova, C. Bourdelle, S. Brezinsek et al., Nucl. Fusion 54, 043001 (2014).

${ }^{8}$ R. Pasqualotto, P. Nielsen, C. Gowers, M. Beurskens, M. Kempenaars, T. Carlstrom, D. Johnson, and JET-EFDA Contributors, Rev. Sci. Instrum. 75, 3891 (2004).

${ }^{9}$ L. Frassinetti, M. N. A. Beurskens, R. Scannell, T. H. Osborne, J. Flanagan, M. Kempenaars, M. Maslov, R. Pasqualotto, M. Walsh, and JET-EFDA Contributors, Rev. Sci. Instrum. 83, 013506 (2012).

${ }^{10}$ R. Scannell, M. J. Walsh, P. G. Carolan, A. C. Darke, M. R. Dunstan, R. B. Huxford, G. McArdle, D. Morgan, G. Naylor, T. O'Gorman, S. Shibaev, N. Barratt, K. J. Gibson, G. J. Tallents, and H. R. Wilson, Rev. Sci. Instrum. 79, 10E730 (2008).

${ }^{11}$ R. J. Groebner, M. A. Mahdavi, A. W. Leonard, T. H. Osborne, and G. D. Porter, Plasma Phys. Controlled Fusion 44, A265 (2002).

${ }^{12}$ A. Kallenbach, N. Asakura, A. Kirk, A. Korotkov, M. A. Mahdavi, D. Mossessian, and G. D. Porter, J. Nucl. Mater. 337-339, 381 (2005).

${ }^{13}$ R. Simonini, G. Corrigan, G. Radford, J. Spence, and A. Taroni, Contrib. Plasma Phys. 34, 368-373 (1994).

${ }^{14}$ D. Reiter, J. Nucl. Mater. 196-198, 80-89 (1992).

${ }^{15} \mathrm{~S}$. Wiesen, EDGE2D/EIRENE code interface report, JET ITC-Report, http://www.eirene.de/e2deir_report_30jun06.pdf, 2006.

${ }^{16}$ O. Sauter, C. Angioni, and Y. R. Lin-Liu, Phys. Plasmas 6, 2834 (1999).

${ }^{17}$ H. R. Wilson, P. B. Snyder, G. T. A. Huysmans, and R. L. Miller, Phys. Plasmas 9, 1277 (2002).

${ }^{18}$ C. D. Challis, J. Garcia, M. Beurskens, P. Buratti, E. Delabie, P. Drewelow, L. Frassinetti, C. Giroud, N. Hawkes, J. Hobirk et al., Nucl. Fusion 55, 053031 (2015).

${ }^{19}$ C. F. Maggi, S. Saarelma, M. Beurskens, C. Challis, I. Chapman, E. de la Luna, J. Flanagan, L. Frassinetti, C. Giroud, J. Hobirk et al., in Proceedings of the 25th IAEA FEC, Saint Petersburg, Russia, 13-18 October 2014, Paper No. EX/3-3.

${ }^{20}$ E. A. Belli, J. Candy, O. Meneghini, and T. H. Osborne, Plasma Phys. Controlled Fusion 56, 045006 (2014).

${ }^{21}$ C. Giroud, G. P. Maddison, S. Jachmich, F. Rimini, M. N. A. Beurskens, I. Balboa, S. Brezinsek, R. Coelho, J. W. Coenen, L. Frassinetti, E. Joffrin et al., Nucl. Fusion 53, 113025 (2013).

${ }^{22}$ C. Giroud, S. Jachmich, P. Jacquet, A. Jarvinen, E. Lerche, F. Rimini, L. Aho-Mantila, N. Aiba, I. Balboa et al., Plasma Phys. Controlled Fusion 57, 035004 (2015). 
${ }^{23}$ A. Järvinen, M. Groth, M. Airila, P. Belo, M. Beurskens, S. Brezinsek, M. Clever, G. Corrigan, S. Devaux, P. Drewelow et al., in Proceedings of the 21st Conference on Plasma Surface Interactions, Kanazawa, Japan, 26-30 May 2014.

${ }^{24}$ A. Järvinen, M. Groth, A. Huber, S. Jachmich, K. Lawson, M. Lehnen, B. Lipschultz, G. Maddison, S. Marsen, A. Meigs et al., in Proceedings of the 25th IAEA FEC, Saint Petersburg, Russia, 13-18 October 2014, Paper No. TH/P5-34.

${ }^{25}$ P. B. Snyder, R. J. Groebner, A. W. Leonard, T. H. Osborne, and H. R. Wilson, Phys. Plasmas 16, 056118 (2009).
${ }^{26}$ M. N. A. Beurskens, T. H. Osborne, P. A. Schneider, E. Wolfrum, L. Frassinetti, R. Groebner, P. Lomas, I. Nunes, S. Saarelma, R. Scannell et al., Phys. Plasmas 18, 056120 (2011).

${ }^{27}$ M. J. Leyland, M. N. A. Beurskens, L. Frassinetti, C. Giroud, S. Saarelma, P. B. Snyder, J. Flanagan, S. Jachmich, M. Kempenaars, P. Lomas et al., Nucl. Fusion 55, 013019 (2015).

${ }^{28}$ M. N. A. Beurskens, L. Frassinetti, C. Challis, T. Osborne, P. B. Snyder, B. Alper, C. Angioni, C. Bourdelle, P. Buratti, F. Crisanti et al., Nucl. Fusion 53, 013001 (2013). 